\title{
Editorial: Physics and Life
}

Over the years Physical Review Letters has been fortunate to have a series of remote editors who are active in research and at the same time handle manuscripts at the interface between physics and biology. With one of the authors of this editorial (Stephan Grill) stepping down from his PRL position after serving for 5 years to start a new "Physics of Life Institute" in Dresden, PRL is happy to announce the successful recruitment of two young talented scientists as remote editors, Olga Dudko from University of California, San Diego, and Raphaël Voituriez from Sorbonne Université, Paris, who have already started handling living matter manuscripts. Olga's current research covers a range of problems in theoretical biophysics and is animated by the notion that deep physics-based conceptual approaches can encompass living-systems complexity. Raphaël's research covers different topics ranging from statistical physics and stochastic processes to biology inspired physics, from single molecule to cell and tissue scales. In what follows, we would like to step back a bit, and articulate why PRL is an excellent venue for the ever more important field of the "physics of life."

Understanding life is one of the greatest scientific challenges. In a series of public lectures delivered in 1943 in Dublin that were the basis for his famous book What is Life? [1], Erwin Schrödinger posed the following important question: "How can the events in space and time which take place within the spatial boundary of a living organism be accounted for by physics and chemistry?" Since then our understanding of the chemical basis of life is now well developed. In comparison, our knowledge of the physics of life, which should complement this biochemical basis, is less advanced. Yet, we must go further and encompass the material science of animate matter, the complex processes of self-organization, as well as information-theoretical aspects.

Living matter has astonishing physical features we are just beginning to grasp. Many scales are involved: Molecules assemble to form functional machines. Organelles compartmentalize and structure the inside of cells. Cells interact to form tissues. Organs and organisms are shaped by the growth and self-assembly of tissues. At scales beyond single organisms individuals can act in collectives. Some go as far as saying that "life is physics" [2,3]. This may be extreme, but physics, as a scientific discipline concerned with processes in space and time, is well positioned to reveal fundamental principles and "laws" that govern the dynamic self-organization of cells, tissues, organs, and organisms.

Both biologists and physicists like to be generalists, searching for overarching principles and universality. But they take distinct approaches. A biologist reveals generality by embracing the complexity of the living world, and by analyzing a large number of related processes in different systems, stressing common features. A physicist reveals generality by theory, systematic deduction, simplification, and experimental tests. However, physicists may also be daunted by the sheer complexity of living systems, with its many different molecules and structures. This is too bad, in part because biologists may turn to increasingly detailed simulations mimicking living systems that may not provide the understanding that a physics approach can bring.

All over the world, physics is stepping up to meet the challenges of a deeper understanding of life. One way forward is to embrace biology, to describe and understand biological processes as physical processes while at the same time acknowledging the complexity of living systems. Another direction is to realize that living matter provides abundant food for thought and stringent tests for the theory of systems out of equilibrium, a theory that might, in turn, help unravel the organizing principles of life.

In any case, physics is deeply involved. Therefore, Physical Review Letters and the other Physical Review journals are involved. We have published important articles on active and living matter ever since the dawn of this field. In the context and the spirit of the evolutions described above, we invite the community to submit their excellent work to PRL, at a time when it is increasingly exciting to work at the physics-biology interface. We hope to receive, and to 
publish, many more important manuscripts identifying fundamental physical mechanisms that underlie and unify complex biological phenomena. And what better way to welcome Olga and Raphaël, our two new editors, than by submitting your best work to them?

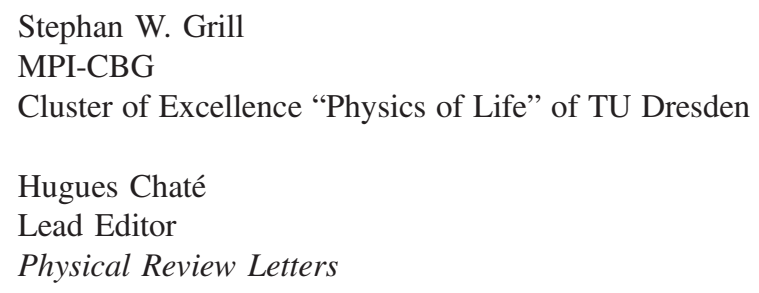

Published 26 September 2019

DOI: 10.1103/PhysRevLett.123.130001

[1] E. Schrödinger, What Is Life? The Physical Aspect of the Living Cell (Cambridge University Press, Cambridge, England, 1944).
[2] N. Goldenfeld and C. Woese, Life is physics: Evolution as a collective phenomenon far from equilibrium, Annu. Rev. Condens. Matter Phys. 2, 375 (2011).

[3] K. Wright, Life is physics, Physics 12, 2 (2019). 